\title{
Enquête
}

Archives de la revue Enquête

5 | 1989

Biographie et cycle de vie

\section{La mobilité sociale entre générations et le « besoin social » de l'éducation}

dans les couches salariées en Finlande après la Seconde Guerre mondiale

\section{Marja Järvelä}

\section{CpenEdition}

\section{Journals}

Édition électronique

URL : http://journals.openedition.org/enquete/85

DOI : 10.4000 /enquete.85

ISSN : 1953-809X

Éditeur :

Cercom, Éditions Parenthèses

Édition imprimée

Date de publication : 2 mars 1989

Référence électronique

Marja Järvelä, «La mobilité sociale entre générations et le « besoin social » de l'éducation », Enquête [En ligne], 5 | 1989, mis en ligne le 27 juin 2013, consulté le 02 mai 2019. URL : http:// journals.openedition.org/enquete/85; DOI : 10.4000/enquete.85 


\title{
La mobilité sociale entre générations et le «besoin social » de l'éducation
}

dans les couches salariées en Finlande après la Seconde Guerre mondiale

\author{
Marja Järvelä
}

1 L'objet de recherche sur lequel je travaille comprend comme éléments principaux 1) les conditions sociales de la mobilité entre les classes sociales, 2) les tendances aux changements de la division du travail social et 3) la divergence des projets de modes de vie des individus et des familles salariées. Mon projet concerne l'étude des histoires de vie, en ce sens que les conditions générales de la socialisation de la main-d'œuvre, donc des couches salariées, sont étudiées en relation avec certaines notions biographiques ("projet de vie», stade de vie, orientation professionnelle et «direction» de vie). L'analyse de la mobilité sociale aboutit à quelques spécifications sur les «macrogénérations» sociales en Finlande qui ont joué un rôle important depuis la Seconde Guerre mondiale.

\section{Sur les aspects de la mobilité sociale des classes sociales en Finlande de 1945 à 1985}

2 Jusqu'à la Seconde Guerre mondiale, les occupations principales du peuple finlandais étaient l'agriculture et l'économie forestière. Le travail salarié était certes assez répandu déjà avant la Seconde Guerre mondiale (la première période de l'industrialisation s'est accomplie principalement vers la fin du XIX ${ }^{e}$ siècle), mais le nombre des personnes dans les familles des couches populaires qui appartenaient à la classe ouvrière industrielle ne représentait qu'une minorité assez réduite. Presque jusqu'à nos jours, la tradition sociale prédominante des couches populaires est plus basée sur le mode de vie paysan des propriétaires, des petits cultivateurs et des familles demi-salariées de l'économie forestière que sur le mode de vie de salariat urbain. 
3 Les couches moyennes salariées possèdent une tradition encore plus courte et plus modeste. Le nombre de fonctionnaires et d'employés dans les services publics commença à croître surtout au début $\mathrm{du} \mathrm{xx}^{\mathrm{e}}$ siècle. Le nombre de fonctionnaires techniciens et administratifs dans les secteurs industriels et les services privés augmenta ultérieurement. Dans les couches moyennes traditionnelles, le groupe le plus important en nombre était assez facile à définir par ses branches d'études. C'étaient principalement des représentants des "professions libérales » ayant suivi des études de médecine et de droit, et bien sûr des officiers et des prêtres qui avaient eux aussi une position clef dans le développement de la nation dont l'indépendance politique ne fut établie qu'en 1917. Au lieu de former les couches sociales supérieures, la formation universitaire a essentiellement produit des éducateurs et des fonctionnaires. Une partie importante de ce groupe fut dispersée dans le pays et forma ainsi un noyau des couches moyennes salariées traditionnelles non urbaines, très attaché au milieu rural.

4 Ce n'est qu'à partir du début des années 1960 qu'on peut accepter une notion de société de salariat moderne pour caractériser la société finlandaise. C'est alors que les grandes masses commencèrent à s'installer dans des milieux urbanisés où la vie quotidienne ne pouvait plus s'accommoder des traditions rurales. Le grand mouvement migratoire de "seconde industrialisation » fut vécu de façons variées chez les différentes générations adultes d'après guerre. Quant au changement de la division du travail social, la Finlande a fait l'expérience d'une modernisation et d'un renversement exceptionnellement rapide des structures d'occupation.

5 En ce qui concerne la succession des générations, le changement structurel est signalé concrètement par le fait que, au début des années 1980, $80 \%$ de la population active occupait un emploi salarié, mais seulement $50 \%$ des pères ou tuteurs principaux avait une occupation salariée. Ce déplacement est évidemment une "conséquence » d'une diminution importante de la population agricole et forestière qui a touché principalement les petits domaines. Aujourd'hui la proportion des paysans dans la population active est de $10 \%$ environ alors que dans la génération des pères elle était de $40 \%$.

6 Le bouleversement de la division du travail social, d'une part, et l'expansion des couches salariées ouvrières, d'autre part, furent tellement rapides après la guerre que les nouveaux venus de la classe ouvrière eurent à peine le temps de s'organiser en syndicats avant que la classe ouvrière industrielle ne commence à se désagréger à cause des nouveaux changements structuraux. En termes statistiques, seul un tiers des ouvriers industriels sont originaires de la classe ouvrière, un peu plus d'un tiers sont nés dans des familles paysannes. Vue sous l'angle du développement d'un mode de vie ou d'un habitus de classe, la seconde industrialisation n'a pas beaucoup consolidé la tradition sociale des familles ouvrières urbaines.

7 La permanence sociale entre les générations n'est pas plus solide dans les couches moyennes salariées. Pourtant les individus des couches moyennes sont un peu plus souvent d'origine urbaine que ceux de la classe ouvrière industrielle.

\section{Système d'éducation et mobilité sociale}

8 Il a fallu concilier deux grands projets éducatifs pour construire un système d'éducation populaire moderne en Finlande. Le premier fut celui de l'instruction populaire basée sur 
une idée de compromis entre les intérêts de la population paysanne et ceux de la population salariée pour constituer une nation uniforme. Le deuxième fut celui d'une sélection et d'une qualification de salariat pour la classe ouvrière et pour les couches moyennes urbaines.

De cette conciliation assez difficile est né le système uniforme d'éducation primaire et secondaire publique de notre époque, un système qui se présente comme un réseau de mobilité sociale qui tend à réduire l'inégalité entre les classes sociales et les différentes couches salariées.

Les conséquences sociales de ce système mis en œuvre dans les années 1970 ne sont perceptibles qu'aujourd'hui et principalement pour ce qui concerne les plus jeunes générations salariées. Auparavant, une importante sélection sociale vers deux voies distinctes était en vigueur dans les écoles : après quatre années de classe élémentaire uniforme pour tous, la majorité des élèves continuait de deux à quatre années à l'école primaire, devenant ainsi apte à entrer dans des écoles professionnelles. Tandis qu'une minorité de chaque génération entrait dans des écoles publiques ou privées pour cinq à huit années d'études supplémentaires : ceci leur permettait d'accéder aux études qui les destinaient aux professions des couches moyennes ou intellectuelles.

Le nouveau système scolaire a la qualité prédominante de retarder la sélection sociale et de la rendre beaucoup plus compliquée. Ceci correspond à la diversité du besoin en maind'œuvre qualifiée mais permet plus facilement l'égalité des chances pour tous indépendamment de l'origine sociale.

12 Cependant le système d'éducation qui se voulait uniforme se développe déjà en liaison avec de nouvelles fonctions de sélection pour faire des distinctions préliminaires entre les futures positions sociales des élèves. Aujourd'hui l'enjeu est de diversifier les programmes et de rendre les choix plus « individuels ». La première langue étrangère est choisie à l'âge de 9 ans. Les premières sélections pour les classes spéciales (classes d'arts et de musique) se font au même âge. D'autre part on opère à l'école primaire de plus en plus de sélection «négative ", particulièrement dans les grandes villes et dans les grands établissements, par la création de classes spéciales pour les élèves mal socialisés. Cette sélection influe certainement sur la position sociale à venir, et probablement même plus que la sélection " positive ».

13 Pourtant, jusqu'ici, le système public de l'école primaire et secondaire en Finlande, indépendamment de sa structure, a plus contribué à promouvoir la mobilité sociale qu'à perpétuer la division des classes sociales. L'absence d'entraves importantes à la mobilité sociale provient simplement de la multitude des voies de déplacement ouvertes par la rapidité du changement de la division du travail social.

Simultanément, une attitude d'aversion à l'égard de l'école a été beaucoup plus rare dans les couches ouvrières finlandaises que dans la classe ouvrière en Angleterre, étudiée par exemple par Paul Willis. Les couches populaires en Finlande semblent avoir beaucoup estimé, jusqu'ici, l'école publique, l'instruction populaire ainsi que les diplômes officiels. Grâce à l'école publique, les classes populaires jugent possible et légitime la promotion sociale.

15 L'idée d'une égalité des chances offerte par l'éducation et, par suite, les perspectives positives pour la promotion sociale ont eu une grande crédibilité, particulièrement dans les années 1960 et 1970 en Finlande. Ce n'est que récemment que l'on se demande si vraiment l'éducation sera une aide à la promotion sociale "automatique». Le 
changement de point de vue est clairement lié au développement actuel du marché du travail : la masse des emplois en nombre absolu a probablement cessé de monter de façon durable.

\section{Les périodes du développement social}

16 L'analyse des aspects du développement social en Finlande permet de distinguer trois périodes distinctes. Ces périodes expliquent le changement des conditions du développement d'un besoin social d'éducation dans les couches salariées et dans les différentes générations après la Seconde Guerre mondiale.

\section{Première période}

La première période se situe entre les années 1945 et 1959. On l'a souvent décrite comme la période de la "reconstruction » (même si simultanément on construisait de nouveaux logements et de nouvelles industries). Par rapport à notre sujet, cette période est bien à même d'être nommée la "période préliminaire d'une société civile populaire de salariat moderne " où l'on voit déjà les classes sociales et les couches salariées traditionnelles s'organiser et se réorganiser en syndicats et où l'on cherche une balance étatique dans l'opposition des classes sociales traditionnelles entre elles.

18 La balance des forces sociales est basée, alors, non seulement sur une idée d'apaisement entre le patronat et les ouvriers, mais aussi sur un compromis entre les intérêts de la population urbaine et rurale. Les zones rurales ont fait l'expérience d'une phase de reconstruction florissante immédiatement après la guerre ce qui a augmenté la demande de main-d'œuvre. Quasiment toute la population rurale ayant la capacité de travailler a rejoint les corps de travail de reconstruction pour satisfaire les besoins alimentaires et de logement.

Simultanément, les industries de base sont construites et reconstruites. Le réseau des services, plus privé que public, commence à être établi dans les agglomérations importantes. La socialisation des familles salariées à une certaine division du travail social et à la poursuite d'un niveau de vie amélioré, mais diversifié entre couches ouvrières et couches moyennes traditionnelles, s'est passée assez facilement. Les destinées du salariat sont assez nettement établies déjà par le système scolaire qui divise, à l'âge de 10 à 12 ans, les jeunes générations en deux groupes sociaux ayant tous les deux des projets de vie distincts quant au marché de la main-d'œuvre et de leur mode de vie.

\section{Deuxième période}

Pendant la deuxième période, celle des années 1960 et des années 1970 à 1975, ce sont surtout les jeunes générations qui sont mobilisées par la recherche de l'emploi. C'est le temps du grand mouvement migratoire et social. Les équilibres entre les principales classes traditionnelles s'écroulent. La Finlande devient une société où les alternatives petites-bourgeoises vont être fortement dominées par le mode de vie salarié qui se répand en particulier dans les villes. Le nombre des individus diminue dans les familles, les femmes deviennent salariées en masse. Au lieu d'un salaire, deux seront nécessaires dans la famille pour atteindre le niveau de vie « conventionnel ». 
21 En comparaison avec la situation d'aujourd'hui, on peut dire que presque tous les nouveaux citadins réussirent à trouver un emploi à condition d'avoir passé un examen de formation professionnelle. Même beaucoup de ceux qui n'avaient pas de certificat purent trouver un travail. Le taux de $4 \%$ de chômeurs fut considéré comme exceptionnellement grave.

Pourtant il ne faut pas oublier qu'environ 400000 Finlandais émigrèrent en Suède pour y trouver un emploi. De plus, pendant les baisses économiques, l'aggravation provisoire du taux de chômage avait touché les régions du Nord et de l'Est plus que le reste du pays.

Les changements essentiels de structures de la division du travail aboutissent à une mutation dans la relation des forces politiques. La classe ouvrière semi-urbanisée a beaucoup amélioré ses conditions matérielles et reste une force politique importante. Pourtant, le nombre de cadres et d'employés des divers services publics ou privés a augmenté rapidement vers la fin de la période, ce qui leur donne un avantage relatif par rapport à la classe ouvrière. Globalement, la mobilité sociale pendant la deuxième période fut très forte et très notoire aussi bien en termes de mobilité entre générations que de mobilité professionnelle (entre métiers ou emplois).

\section{Troisième période}

La baisse économique de la fin des années 1970 présume déjà de l'évolution future. La proportion de la classe ouvrière industrielle dans la totalité de salariat commence à diminuer. La position sociale des cadres et des employés, dont le nombre augmente rapidement, est au contraire fortifiée. Jusqu'ici ce développement a renforcé aussi le rôle $\mathrm{du}$ gouvernement, devenu l'employeur principal dans notre pays. Les femmes sont de plus en plus nombreuses sur le marché de l'emploi grâce surtout à leur haut niveau d'études (indiqué par le nombre de diplômes officiels). En même temps, l'égalité des chances entre les sexes sur le marché de l'emploi, notamment en terme de mobilité professionnelle, est devenue l'un des thèmes politiques les plus souvent cités. Le nombre de membres de la famille nucléaire n'a cessé de diminuer; en même temps ces familles sont soumises à un contrôle plus immédiat et plus compliqué qu'avant grâce aux politiques sociales.

La durée biographique d'une carrière salariée personnelle commence à diminuer au lieu d'augmenter. La durée des études se prolonge. Les arrangements spéciaux pour la retraite anticipée s'aménagent de plus en plus fréquemment afin d'éviter des situations critiques telles que le chômage et la succession de générations.

Le niveau de vie s'améliore encore dans beaucoup de familles de deux salaires qui ont une position bien établie dans le salariat ouvrier ou dans les couches moyennes. Les biens (comptés en marks) des familles des nouvelles couches moyennes, employées surtout dans le secteur commercial et les industries clef, s'accroissent et l'argent n'est plus consacré uniquement aux besoins de la vie quotidienne. Une partie du salaire est placée à la bourse. Surtout, les nouvelles couches moyennes salariées et les retraités aisés adoptent de nouveaux modèles de mode de vie en combinant différentes situations sociales telles que celles de propriétaire et de salarié (ou ancien salarié). Celles-ci s'écartent irrémédiablement des modes de vie traditionnels des couches moyennes en Finlande, d'un niveau de vie assez modeste. 
L'empreinte du « marché » est présente dans presque toutes les activités et tous les lieux de vie. Une des qualités les plus utiles chez les familles salariées (ou futures salariées) est la connaissance des fonctionnements des différentes sortes de marchés. Sans stratégie individuelle ou familiale, il n'y a pas de succès possible sur le marché du travail qualifié. Le diplôme ne garantit plus qu'une entrée légitime dans la compétition.

\section{Types de stratégies chez les couches salariées}

Pour conclure, il m'est apparu qu'une certaine typologie sociale était possible pour la population salariée relativement aux choix offerts par le système scolaire et par la diversité des chances sur le marché du travail. Il s'agit d'une typologie où la population est divisée en trois groupes caractérisés par le type de méthode adoptée face au marché du travail et face à la stratégie d'orientation sociale.

Le premier type de stratégie sera celui de la classe ouvrière conçu comme le «type de base » parmi ces stratégies sociales des couches salariées. Le deuxième type est celui formé par des couches moyennes modernes très variées. Le troisième est celui de la classe moyenne salariée traditionnelle, incluant la classe intellectuelle salariée.

Un modèle idéal de l'orientation sociale dans les principales couches salariées

\begin{tabular}{|c|c|c|c|}
\hline & Clesse ourvilere & Nouvales couches moyennes: & Couches moyernes traditorneles \\
\hline Orientation salandbo & $\begin{array}{l}\text { assurance de scutce } \\
\text { de revenus }\end{array}$ & $\begin{array}{l}\text { mobilide probessionnelle et } \\
\text { mobiline entre genérations }\end{array}$ & $\begin{array}{l}\text { comperences sociale et inteliectuelle } \\
\text { ganeralisees. }\end{array}$ \\
\hline Qualification & qualification de base salarièe & $\begin{array}{l}\text { élasticité de qualification } \\
\text { professionnele }\end{array}$ & cognition abstraite \\
\hline Type d'budes suvies & $\begin{array}{l}\text { education de base ou } \\
\text { de niveau ordinaire }\end{array}$ & $\begin{array}{l}\text { avantage relatif par diphome } \\
\text { et par cours spéciaux }\end{array}$ & $\begin{array}{l}\text { association bicgraphique (education, } \\
\text { travai et style de vie) }\end{array}$ \\
\hline
\end{tabular}

Ce classement en trois stratégies me semble avoir une certaine stabilité explicative par rapport à des divisions essentielles de besoin social d'éducation parmi les couches salariées en Finlande dans l'époque d'après-guerre.

31 Non seulement les différentes couches sociales traduisent des orientations variées dans leurs intérêts pour l'éducation, mais de plus l'expérience sociale du marché du travail à l'égard des "places vides » a varié, conformément aux positions sociales d'origine, par génération et par sexe.

\section{BIBLIOGRAPHIE}

AHPONEN P., JÄRVELÄ M. - « The changing way of life as a challenge to workers and the worker community ", in J. P. Roos \& Andrzej Sicinski, eds, Ways of Life in Finland and Poland. Comparative Studies on Urban Populations, Aldershot-Brookfield, VT, Avebury, 1987.

AHRne, G. et al., eds - Class and Social Organisation in Finland, Sweden and Norway, Uppsala, Academia Upsaliensis, 1988 (« Acta Universitatis Upsaliensis. Studia sociologica Upsaliensia » 28). 
BALLION, R. - Les consommateurs d'école. Stratégies éducatives des familles, Paris, Stock, 1982 (« StockLaurence Pernoud »).

BERTAUX, D. - Destins personnels et structures de classes, Paris, Presses universitaires de France, 1977. BOLTANSKI, L. - Les cadres. La formation d'un groupe social, Paris, Minuit, 1982.

BouRdieu, P. - La distinction. Critique sociale du jugement, Paris, Minuit, 1979. - « Espace social et genèse des "classes" ", Actes de la Recherche en Sciences sociales, 52-53, juin 1984, p. 3-12.

JÄRVELÄ, M. - « The permanency of social classes and intergenerational mobility in Finland. Research note ", Acta sociologica. Journal of the Scandinavian Sociological Association, 26 (3-4), 1983, p. 287-298.

PASSERON, J.-C. - « L'inflation des diplômes. Remarques sur l'usage de quelques concepts analogiques », Revue française de Sociologie, 23 (4), oct.-déc. 1982, p. 551-584.

Roos, J. P., RAHKONEN, K. - «In search of the Finnish new middle class ", Acta sociologica. Journal of the Scandinavian Sociological Association, 28 (3), 1985, p. 257-274.

WILLIS, P. - Learning to Labour. How Working Class Kids Get Working Class Jobs, Londres, Saxon House, 1977. 